\title{
Influencia de variables meteorológicas sobre el rendimiento de la caña de azúcar
}

Influence of meteorological variables on sugarcane yield

Sieiro-Miranda, Grethel L. ${ }^{1 凶}$, González-Hidalgo, Maribel ${ }^{1}$, Lozano-Rodríguez, Miguel

Á. ${ }^{2}$, Raya-Cruz, Blanca E. ${ }^{2}$, González-Salas, Miguel Á. ${ }^{2}$ y Aguirre-López, Enrique ${ }^{3}$.

${ }^{1}$ Instituto de Investigaciones de la Caña de Azúcar (INICA). Carretera CUJAE km. 1²/2, Boyeros, La Habana, Cuba. Teléfonos: (537) 260 2571, (537) 262 4436-37. ${ }^{2}$ Facultad de ciencias Biológico Agropecuarias campus Tuxpan. Universidad Veracruzana. ${ }^{3}$ Facultad de ciencias Agrícolas campus Xalapa. Universidad Veracruzana.

${ }^{凶}$ Autor para correspondencia: grethel.sieiro@inica.azcuba.cu

Recibido: 03/01/2016

Aceptado: 10/06/2016

\section{RESUMEN}

Se seleccionaron 14 ingenios azucareros pertenecientes a las provincias Artemisa, Mayabeque y Matanzas, con sus respectivas estaciones meteorológicas asociadas. Los datos anuales de rendimiento agrícola e industrial de la caña de azúcar y los registros diarios de precipitaciones y temperaturas en el período comprendido entre 1980 y 2011 (30 años), sirvieron de base para el estudio. El análisis de los datos arrojó que en todo el período, la temperatura máxima (Tmáx) no experimentó cambios, la temperatura media (Tm) aumentó en $0,93^{\circ} \mathrm{C}$, mientras que la temperatura mínima (Tmín) lo hizo en $0,62^{\circ} \mathrm{C}$ y la amplitud térmica (AT) se redujo en $0,72^{\circ} \mathrm{C}$. De todas las regiones estudiadas, la estación meteorológica de Bahía Honda fue la que mostró los menores valores de AT. Las precipitaciones incrementaron en $33,78 \%$, mientras que RA disminuyó en $52,83 \%$ con relación a su valor inicial. Se encontró una dependencia inversa entre la AT y la Tmín, mientras que hubo una fuerte dependencia entre RI y Prec (97\%) en las tres provincias. Estas últimas, 10 días antes de la cosecha, tuvieron un ligero efecto negativo sobre RI, en tanto que, éste no fue prácticamente afectado. Se concluyó que la provincia Matanzas fue la que presentó las mejores condiciones climáticas para el cultivo de la caña de azúcar y dentro de ella, la zona más propicia para su desarrollo fue el municipio Jovellanos.

Palabras claves: rendimiento agrícola, rendimiento industrial, amplitud térmica 


\begin{abstract}
The study was setting up in fourteen sugar mills from Artemisa, Mayabeque and Matanzas provinces, with its associated meteorological stations. Annual sugarcane field yield (RA) and Industrial yield (RI), besides records of monthly maximum temperature (Tmax), minimum (Tmin), (Tm) and precipitation (Prec) in the period between 1980 and 2011 (30) were the basis for this research. The data analysis revealed that throughout the period, Tmax was unchanged, $\mathrm{Tm}$ increased in $0,93^{\circ} \mathrm{C}$ while Tmin did in $0,62^{\circ} \mathrm{C}$ and thermic oscillation (AT) fell $0,72^{\circ} \mathrm{C}$. Meteorological station of Bahia Honda displayed the lowest AT value. The rainfall increased in $33.78 \%$, while RA decreased $52.83 \%$ compared to its initial value. An inverse dependence between AT and Tmin was found, while there was a strong dependence between RI and Prec (97\%) in all provinces. These last 10 days before harvest, had a negative effect on RI, while this was not practically affected. It was concluded that Matanzas was the province with the best climate conditions for sugarcane cropping and within the area the most favorable for its development was Jovellanos.
\end{abstract}

Keywords: Agricultural yield; industrial yield, thermal amplitude, Cuba

\section{INTRODUCCIÓN}

La caña de azúcar (Saccharum spp.) fue una de las primeras plantas tropicales en ser adaptada a condiciones de cultivo a gran escala. Es una planta $\mathrm{C} 4$, altamente productora de biomasa, apta para fijar grandes cantidades de dióxido de carbono ( ) (PonceHernández y col., 2004). Actualmente se produce comercialmente en aproximadamente 70 países, con casi 22 millones de hectáreas cosechadas cada año entre las latitudes $35^{\circ} \mathrm{N}$ y $35^{\circ} \mathrm{S}$ (Cerri y col., 2010). En Cuba este cultivo ocupa alrededor de 800 mil hectáreas y crece y se produce de manera intensiva, en una amplia gama de suelos, distribuidos a lo largo y ancho de la isla. La región occidental de Cuba presenta variabilidad climática respecto a la precipitación y la temperatura, lo que determina en parte que el territorio cuente con diferentes grados de aptitud para el cultivo de la caña de azúcar. El clima, así como los suelos de la región, son determinantes en el crecimiento y desarrollo de la caña de azúcar (Herrera, 2000). En esta región la caña de azúcar ocupa aproximadamente
106052.70 ha, con un RA medio que ha variado desde 1981 hasta 2010 en 25.19 $\mathrm{t} \mathrm{ha}^{-1}$, alcanzando en $2010,34.05 \mathrm{t} \mathrm{ha}^{-1}$. La capacidad de la planta de caña de azúcar para producir sacarosa (azúcar comercial) depende de la variedad, el manejo agronómico del cultivo y de factores climáticos. Los principales componentes climáticos que controlan el crecimiento, el rendimiento y calidad de la caña son la temperatura, la luz y la humedad disponible. La connotación que tenga un factor climático dependerá de la región en que se desarrolle, por la intensidad o duración con que este se manifieste. En Cuba, los factores limitantes para el crecimiento y desarrollo de la caña de azúcar son la temperatura y la precipitación (Herrera, 2000).

La caña de azúcar es un cultivo que difiere de otros en cuanto a sus exigencias en humedad y temperatura. En los primeros estadíos, requiere de humedad adecuada y temperaturas cálidas y en la etapa de maduración, por el contrario, es beneficiada por bajas temperaturas y una disminución 
apreciable de la humedad, lo que es indispensable para un rendimiento azucarero óptimo, siendo los rendimientos agrícolas e industriales que seobtienen en un lugar determinado, el resultado de la interacción de elementos edáficos, climáticos y del manejo del cultivo. Las temperaturas bajas son determinantes en la formación de sacarosa, siendo particularmente importante que prevalezca una diferencia acentuada entre las máximas y mínimas, para una adecuada acumulación de sacarosa (Solano, 2014). En Cuba se conoce que la disminución en la AT, afecta a cultivos de importancia económica, como el tabaco, con una influencia negativa en el contenido de clorofila de las hojas y una aceleración en la madurez y la senescencia (INSMET, 2012), aspecto este que no ha sido estudiado para el cultivo de la caña de azúcar. Teniendo en consideración la incidencia determinante de los factores climáticos sobre la producción de caña de azúcar, se planteó como objetivo, determinar la influencia de variables meteorológicas en el rendimiento de la caña de azúcar en las provincias Artemisa, Mayabeque y Matanzas.

\section{MATERIALES Y MÉTODOS}

Para el estudio se seleccionaron empresas azucareras pertenecientes a las provincias Artemisa, Mayabeque y Matanzas. De la región se tomaron 14 Uniones Empresariales de Base (UEB), que representan más del 9,5\% del área dedicada a la producción azucarera del país (SERFE, 2015).

Los datos meteorológicos, brindados por el Instituto de Meteorología (INSMET, 2015), comprendieron registros diarios de Prec y temperaturas, Tmax y Tmín, asociados a los pluviómetros ubicados a no más de $30 \mathrm{~km}$ del radio de influencia de la empresa. Basándonos en este criterio se escogieron 10 estaciones meteorológicas. Del cultivo se tomó el RA y el RI, ambos expresados en $\mathrm{t} \mathrm{ha}^{-1}$ de cada una de las Unidades Empresariales de Base (UEB), correspondientes al período 1981-2014. Estos datos pertenecen a la base patrimonial del Instituto de Investigaciones de la Caña de Azúcar (INICA).

Con ambas fuentes, se conformó una gran base de datos, que comprendió las provincias Artemisa, Mayabeque y Matanzas, en el período 1981-2010. La base de datos estuvo conformada por el RA e RI ( $\left.\mathrm{tha}^{-1}\right)$; fecha de plantación y corte; Tmax y Tmín (grado Celsius); Prec (mm). A partir de estas se calcularon las variables, Tm, AT, AT acumulada (ATacc), y los grados días (GDD).

La AT fue calculada con la utilización de las temperaturas máximas y mínimas, mediante la ecuación (1): (1) Dónde: AT es la amplitud térmica; Tmáx es la temperatura máxima y Tmín es la temperatura mínima.

Los GDD fueron calculados de acuerdo con Jiménez y col. (2004), utilizando la ecuación (2).GDD $=\sum$ (2)

Dónde: Tmáx es la temperatura máxima; Tmín es la temperatura mínima; Tbase es la temperatura por debajo de la cual, se detiene el crecimiento. Se realizó la caracterización climática de cada una de las tres provincias occidentales, teniendo en cuenta la media mensual de las Prec, la Tmax y la Tmín. 
Se construyó una gran base de datos con todos los campos georeferenciados con el objetivo de llevar al plano geográfico la variabilidad de las variables meteorológicas y su relación con los rendimientos. Para representar en el plano geográfico los rendimientos $\mathrm{y}$ las precipitaciones por provincia, se utilizó el sistema de información geográfica MAPINFO, para lo que se preparó una base de datos georeferenciada por provincia y estación meteorológica.

El procesamiento estadístico de los datos se realizó con la utilización del programa Statistica versión 8. Una vez comprobada la normalidad de los datos mediante la prueba de Shapiro Wilk W, se procedió a la comparación de medias a través de análisis de varianza a 5\% de probabilidad de error y en caso de no cumplirse el criterio de normalidad, se aplicaron pruebas no paramétricas.

\section{RESULTADOS Y DISCUSIÓN}

Al comparar las variables climáticas en las tres provincias occidentales, se observó un comportamiento diferenciado entre ellas (Figura 1). Correspondieron a Mayabeque las mayores Prec y las Tmín más bajas. Matanzas, ostentó las menores Prec y las Tmax más altas y Artemisa, la Tmín más alta de las tres.

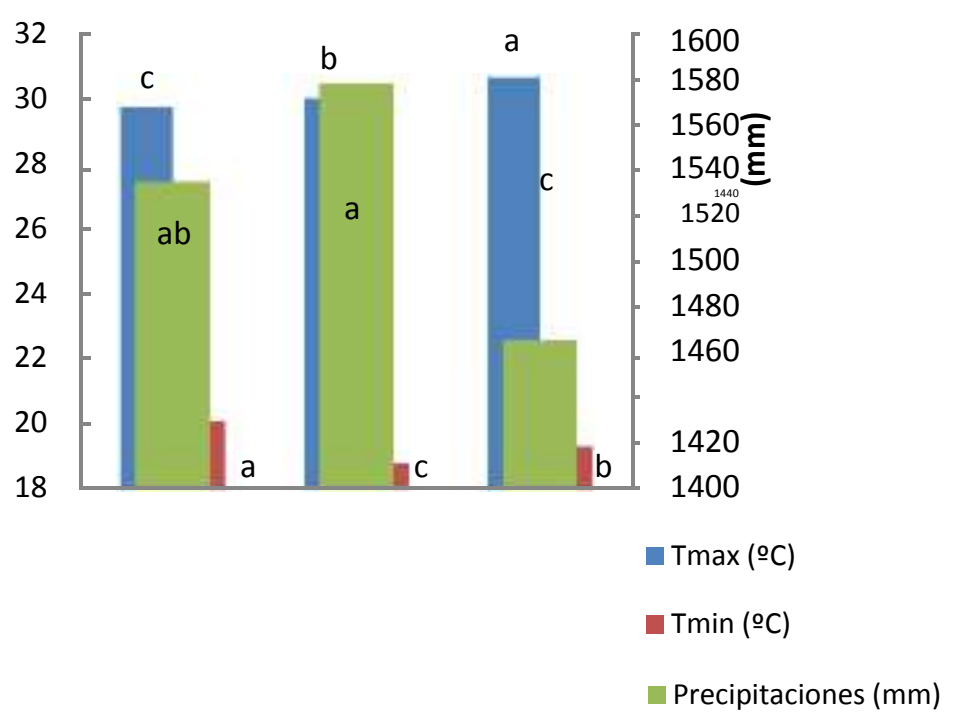

Figura 1. Comportamiento de las Tmín, Tmax y Prec en el área de estudio en el período 1981-2010. Letras desiguales expresan diferencias estadísticamente significativas a 5\% de probabilidad de error, entre los valores de cada provincia.

Los valores de Tmín y Prec encontrados en la provincia Artemisa, pudieron estar influidos por el comportamiento de estas variables en la estación meteorológica de Bahía Honda. De manera general, los menores valores de esta variable se registraron en Artemisa, seguida de Mayabeque y Matanzas, lo que demostró que, en la medida en que avanzamos hacia el Este, hubo una tendencia a su incremento, lo que corrobora lo planteado por Lecha y col. (1994) quienes plantearon que existe un ligero aumento de la temperatura del aire en sentido Oeste a Este. Las variaciones encontradas en el clima en la región occidental en 30 años, es una prueba de que, en Cuba, el clima está cambiando. Los vaticinios de INSMET (2012) en su informe al Panel Intergubernamental de Cambio 
Climático (IPCC), en Cuba, expresan que las Tm aumentarán en 1,4 a $2,0^{\circ} \mathrm{C}$ y que las Prec tendrán un decrecimiento de 20 y $50 \%$ para el año 2050. En este estudio se encontró un aumento incremento de la Tmín y de las Prec en áreas asociadas al cultivo de la caña de azúcar.

Al analizar la AT en cada una de las estaciones meteorológicas en el período 1981-2010, se observó que la correspondiente a Bahía Honda, mostró el menor valor en relación con el resto. Si se compara con el rango óptimo publicado por Larrahondo y Villegas (1995) de $11-13^{\circ} \mathrm{C}$, la cifra reportada aquí fue muy inferior $\left(8,1^{\circ} \mathrm{C}\right.$ ) (Figura 2$)$, lo que puede deberse a la cercanía al mar del lugar, que es uno de los factores moduladores de la oscilación térmica.

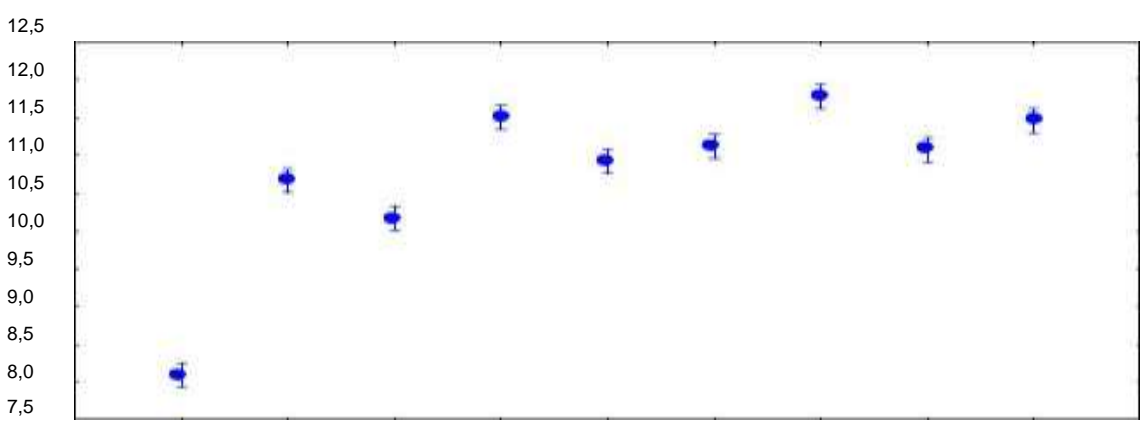

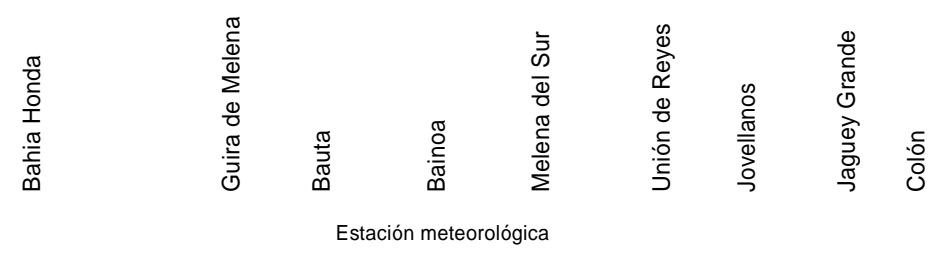

Figura 2. AT en las diferentes estaciones meteorológicas asociadas a las áreas cañeras de las provincias Artemisa, Mayabeque y Matanzas en el período 1981-2010. Barras verticales indican intervalos de confianza a un nivel de significación de 5\%.

Cuando se relacionó la AT con la Tmín en la región occidental, se observó una disminución en el período $1981-2010$, que varío de $11,11^{\circ} \mathrm{C}$ en 1981 a $10,38^{\circ} \mathrm{C}$ en 2010 . Este decrecimiento estuvo asociado al aumento de las Tmín (Figura 3), donde la variabilidad encontrada en AT fue explicada en $69 \%$ por la Tmín, mediante la ecuación: AT $\left({ }^{\circ} \mathrm{C}\right)=$ 30,2434-1,00*Tmín $\left({ }^{\circ} \mathrm{C}\right) ; \mathrm{R}^{2}=0,69$. Es decir, por cada grado que aumente la Tmín, la AT disminuirá en un grado Celsius. Válido solamente para el rango de temperaturas y AT que se muestra en la Figura 3.

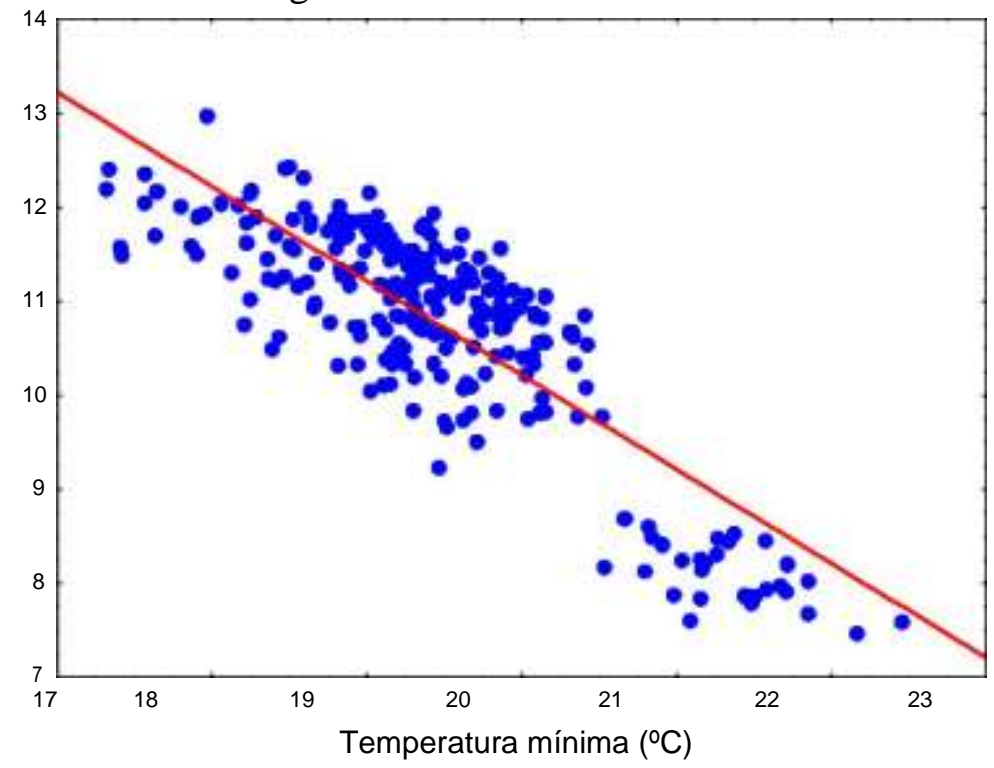


Figura 3. Relación entre la AT y la Tmín en las provincias Artemisa, Mayabeque y Matanzas (1981-2010).

En la Tabla 1 se observa el porcentaje de la frecuencia absoluta por intervalo de AT. La categoría $9-11^{\circ} \mathrm{C}$ fue la de mayor frecuencia, representad por la provincia Matanzas, seguida de Artemisa y en último lugar, Mayabeque, provincia esta que ostentó la mayor frecuencia en el intervalo $11-13^{\circ} \mathrm{C}$, que según Larrahondo y Villegas (1995) para condiciones tropicales, como las de
Cuba, la diferencia entre las Tmax y Tmín oscilante entre 11 y $12^{\circ} \mathrm{C}$, estimula el almacenamiento de sacarosa. Este resultado serviría como un criterio a tener en consideración, para direccionar la producción cañera hacia aquellos lugares donde la AT es la adecuada para obtener mayores producciones.

Tabla 1. Porcentaje por rango de frecuencia de la AT en las provincias occidentales.

\begin{tabular}{llll}
\hline \multirow{2}{*}{ Rango } & Artemisa & Mayabeque & Matanzas \\
\cline { 2 - 4 } & & $\%$ & \\
\hline 2,69 & 0,14 & 0 \\
& 33,18 & 4,86 & 3,19 \\
44,67 & 41,11 & 43,06 \\
17,61 & 42,92 & 37,99 \\
1,85 & 10,00 & 14,79 \\
0,00 & 0,97 & 0,97 \\
\hline
\end{tabular}

Si se tiene en cuenta la distribución de frecuencias presentada en la Tabla 2, de acuerdo con Larrahondo y Villegas (1995), la zona más propicia para el desarrollo de la caña de azúcar sería el municipio de Jovellanos, de la provincia Matanzas, que agrupa las áreas asociadas a las UEB Jaime López, Julio Reyes, Granma, Rabí y Smith Comas, ya que la misma presentó todos los valores de AT en el rango recomendado, lo que explica su menor tasa de variación en los rendimientos (Tabla 3).

Tabla 2. Frecuencia absoluta de AT por estación meteorológica.

\begin{tabular}{|c|c|c|c|c|c|c|c|c|c|}
\hline \multirow{2}{*}{ Rango } & \multicolumn{9}{|c|}{ BH $\quad$ GM $\quad$ BT $\quad$ BA $\quad M S$ UR JV JG } \\
\hline & \multicolumn{9}{|c|}{ No. de observaciones } \\
\hline $7 \leq \mathrm{AT}<9$ & 30 & 0 & 0 & 0 & 0 & 0 & 0 & 0 & 0 \\
\hline $9 \leq \mathrm{AT}<11$ & 0 & 6 & 29 & 6 & 18 & 12 & 0 & 14 & 4 \\
\hline $11 \leq \mathrm{AT}<13$ & 0 & 24 & 1 & 24 & 12 & 18 & 30 & 16 & 26 \\
\hline
\end{tabular}

La Tabla 3 representa la tasa de variación anual de las variables asociadas al clima y al rendimiento en las tres provincias occidentales durante 30 años. 
Tabla 3. Tasas de variación anual de las variables climáticas asociadas a las áreas cañeras de las tres provincias occidentales.

\begin{tabular}{lllll}
\hline & \multicolumn{1}{c}{ Unidades } & Artemisa & Mayabeque & Matanzas \\
\hline Tmáx & ${ }^{\circ} \mathrm{C}$ año & \\
Tmín & & $-0,035$ & $-0,032$ & $-0,016$ \\
& & $-0,040$ & $-0,030$ & $-0,045$ \\
\hline Tmed & & & & \\
\hline AT & mm año-1 & $-0,042$ & $-0,054$ & $-0,019$ \\
\hline Prec & & $-13,004$ & $-0,001$ & 0,029 \\
\hline Rend. Agt ha-1 año-1 & 1,34 & $-8,043$ & $-8,359$ \\
\hline
\end{tabular}

El signo negativo (-) significa incremento, el signo más (+), decrecimiento.

Se apreció una disminución anual en RA, que fue dos veces superior en Artemisa y Mayabeque que en Matanzas, este comportamiento pudiera estar asociado a los mayores incrementos en la tasa de variación de la Tmax en las dos primeras provincias y menor en la última, además de presentar el máximo de frecuencias en el rango óptimo de AT, comparada con las demás provincias. La tasa de incremento para la Tmín fue superior en Matanzas, seguido de Artemisa y Mayabeque. En cuanto a la variación anual de la AT, la mayor disminución fue observada en Matanzas. Con relación a la variación anual de las precipitaciones, se observó un incremento con el decurso del tiempo en las tres provincias, con el mayor valor en la provincia Artemisa.

Se apreció un decrecimiento del rendimiento con el tiempo, que se ajustó a un modelo cuadrático (Figura 4). La variación experimentada fue de $67,17 \mathrm{t}$ caña $\mathrm{ha}^{-1}$ en 1981 a $35,49 \mathrm{t}$ caña $\mathrm{ha}^{-1}$ en 2010, con una diferencia entre ambos extremos de 52,83\%. Este comportamiento fue común para las tres provincias, al no encontrarse diferencias estadísticamente significativas al 5\% entre ellas $[\mathrm{F} \quad(58,180)=0,91164$, $\mathrm{p}=0,65256]$.

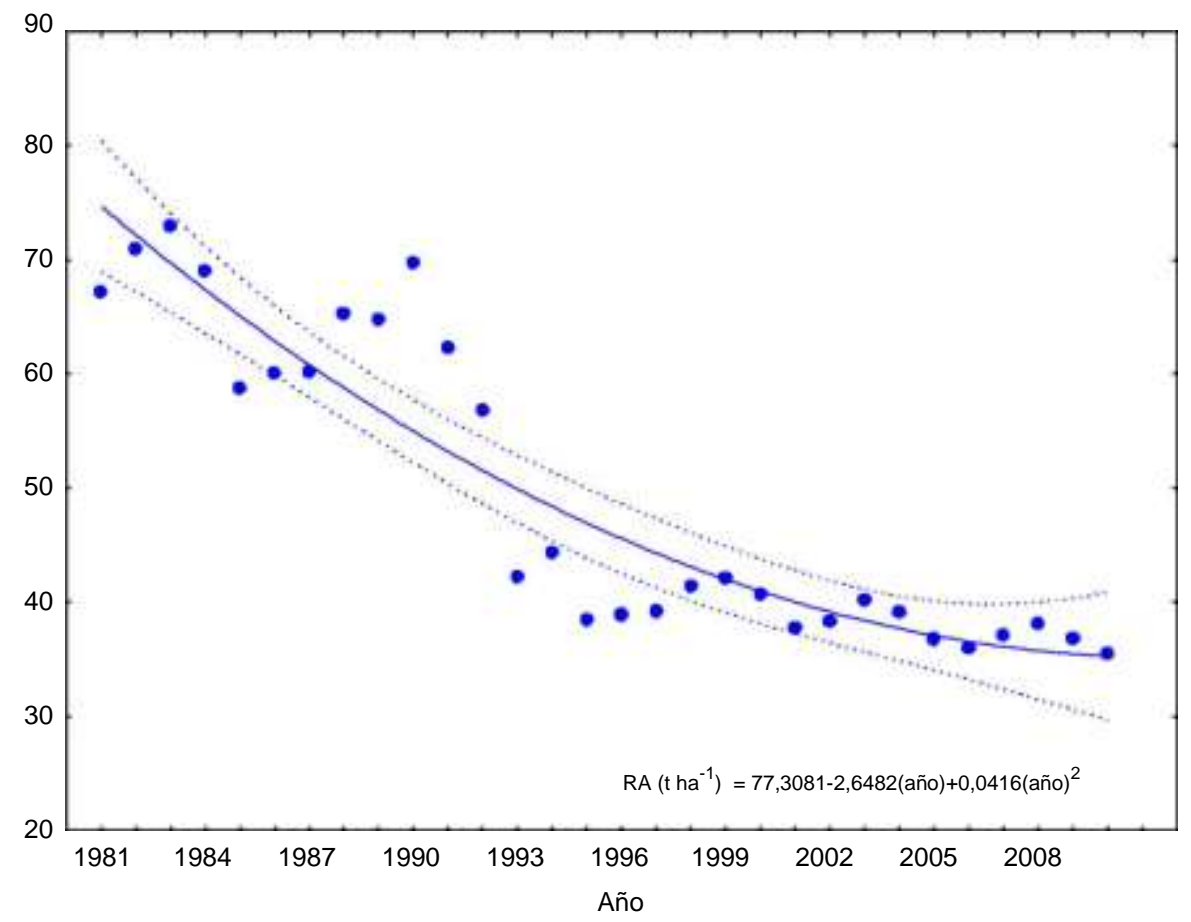

Figura 4. Comportamiento general del rendimiento agrícola en las provincias Artemisa, Mayabeque y Matanzas en el período comprendido entre 1981 y 2010, ambos inclusive. Las bandas significan el intervalo de confianza a 0,95 
Esta conducta alerta sobre la contrarrestar la declinación de los necesidad de tomar medidas en aras de rendimientos, siendo necesario, profundizar en las causas que con llevan a ello. Al respecto, autores como González y col. (2010) plantearon la necesidad de valorar un cambio en las prácticas de manejo a los suelos cañeros y añadieron que la labranza convencional, unida al monocultivo, son los responsables de la declinación de la fertilidad de los suelos, debida a la pérdida de materia orgánica por oxidación y con ello, de su productividad.

Es necesario tener presente que las áreas analizadas son cultivadas en secano, razón que se suma a las causas expuestas en párrafo precedente y que tiene gran incidencia en los bajos rendimientos del cultivo, por no permitir que manifieste su máximo potencial productivo (Reynoso, 1962).

Con relación al RI, el comportamiento fue similar al RA, pero no tan seriamente afectado como este último, con una diferencia con el valor inicial de $1,59 \mathrm{t} \mathrm{ha}^{-1}$ de azúcar, al término de 30 años. La disminución experimentada en RA y RI, está asociada a factores diversos, entre los que se pueden mencionar, el manejo, el tipo de suelo, las necesidades hídricas y la interacción con el clima.

Se obtuvo una relación directa entre el rendimiento y las Prec en las tres provincias estudiadas (Figura 5), donde las Prec explicaron $97 \%$ de la variabilidad del RI. Investigaciones hechas por Castro y Suárez (2011) en Guatemala, encontraron una relación significativa entre estos dos parámetros, con un coeficiente de determinación que explicó entre 74 y $75 \%$ la varianza del rendimiento. De igual manera, van

Dillewijn (1952), encontró un comportamiento similar al relacionar ambas variables

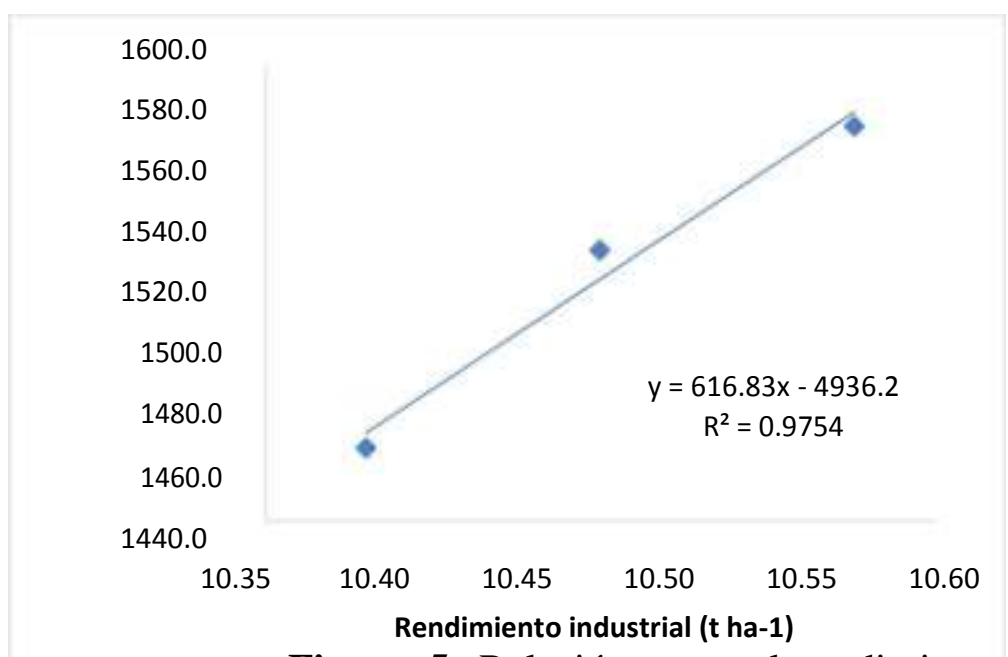

Figura 5. Relación entre el rendimiento industrial y las precipitaciones en las provincias Artemisa, Mayabeque y Matanzas.

En la provincia Matanzas se encontró una tendencia inversa entre el RA y las precipitaciones 10 días ( $\mathrm{p}-10 \mathrm{dac}$ ) antes de la cosecha, que se ajustó a un modelo exponencial de la forma $\mathrm{RA}\left(\mathrm{t} \mathrm{ha}^{-1}\right)=$ $58,1543 * \exp (-0,022 * \mathrm{p}-10 \mathrm{dac})$ 
donde p-10 dac, explicaron $60 \%$ de la variabilidad de los rendimientos $(\mathrm{r}=$ $0,6043 ; \mathrm{p}=0,0048)$.

La Figura 6 muestra la relación entre el RA y los GDD en el período comprendido entre la plantación y la cosecha (zafra). Se advierte que ambas variables tendieron a disminuir en el tiempo, no encontrándose una relación directa, debido a la variabilidad presentada. Con relación a este aspecto, Robertson y Thorburn (2007) encontraron una dependencia directa entre GDD y la productividad del cultivo.

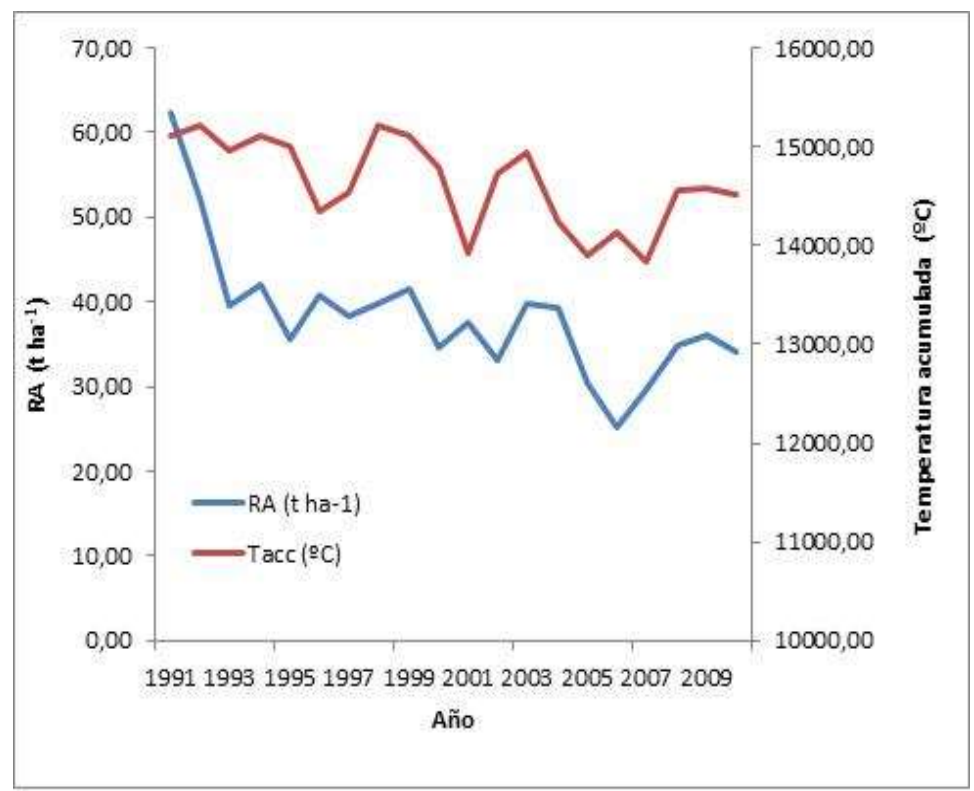

Figura 6. Relación entre el rendimiento agrícola (RA) y GDD en las provincias Artemisa, Mayabeque y Matanzas (1981-2010). (RI es rendimiento industrial; AT es amplitud térmica; GDD son los grados días).

En la medida en que aumentó la edad, también lo hizo el RA, lo que estuvo en correspondencia con el incremento de biomasa en la medida en que la planta envejeció, reparando en que esto sucede hasta un punto en que la planta madura y cesa su crecimiento. Este comportamiento se ajustó a un modelo logarítmico (Figura 7). 


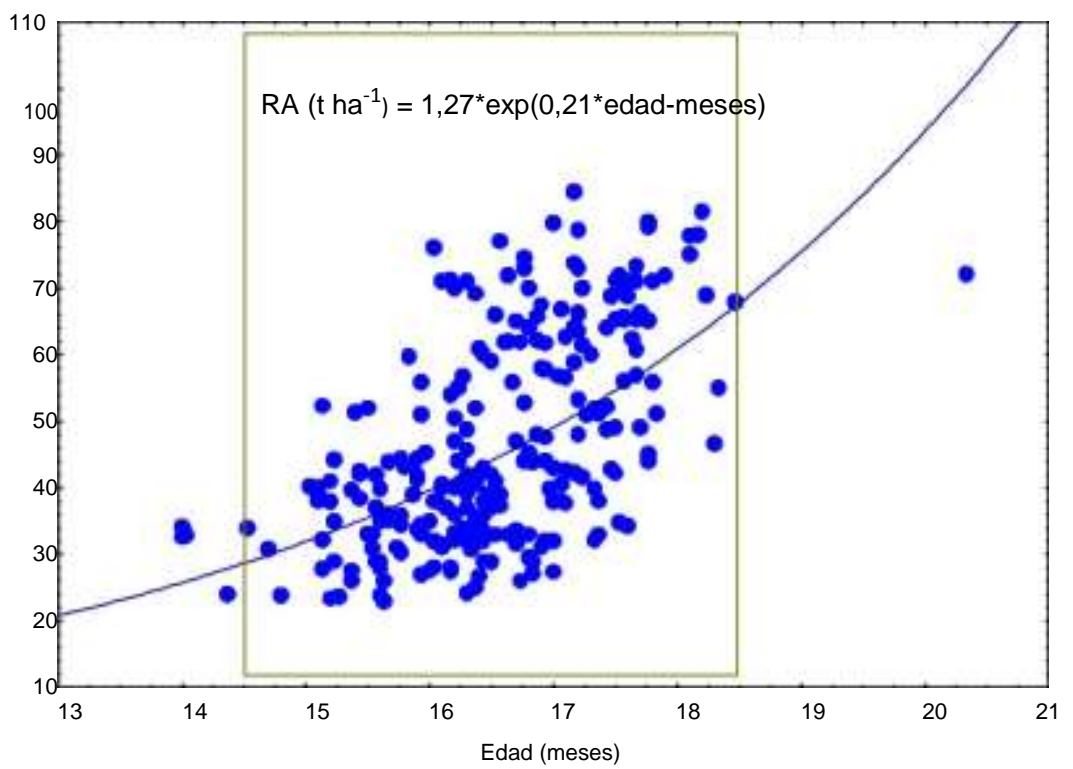

Figura 7. Relación entre el RA de la caña de azúcar en la provincia Matanzas en el período 1981-2010.

\section{CONCLUSIONES}

- $\quad$ Las temperaturas cardinales y las Prec incrementan en el período 1981-2010.

- $\quad$ La AT tiende a disminuir durante el periodo 1981-2010, siendo la estación meteorológica de Bahía Honda la que menores valores ostenta, comparada con el resto de la zona cañera estudiada.

- Se encuentra una dependencia inversa entre la AT y la Tmín y directa entre RI y las Prec en Artemisa, Mayabeque y Matanzas.

- $\quad$ El RA disminuye en 52,83\% con relación a su valor inicial, mientras que el RI, no se afecta.

- Se encuentra una tendencia a la disminución a partir de 1991 en el RA y los GDD.

- La provincia Matanzas es la que presenta las mejores condiciones climáticas para el desarrollo de la caña de azúcar y dentro de ella, el municipio Jovellanos.

\section{LITERATURA CITADA}

Castro, O. y Suárez, A. 2011. La
Azúcar. En CENGICAÑA: El cultivo de la caña de azúcar en

Guatemala. CENGICAÑA.

Cerri C.C.; Galdós M.V.; Maia S.M.F.; Bernoux M.; Feigl B.J.; Powlson D. y Cerri C.E.P. 2010. Effect of sugarcane harvesting systems on soil carbon stocks in Brazil: an examination of

existing data. European Journal of Soil Science, doi: 10.1111/j.1365-

2389.2010.01315.x.

González H. Maribel; de León M.E; Villegas R.; Pineda E.; Cortegaza P.L. Pablos P. y Angarica E. 2010. Influencia del manejo de la caña de azúcar sobre las cualidades del suelo y el rendimiento

agrícola. Revista Cuba\&Caña. ISSN 1028-6527. No 2, p. 13-20.

Herrera, M.S. Contribución metodológica a la zonificación agroclimática de

la caña de azúcar: Caracterización agroclimática de las áreas cañeras de la 
provincia La Habana. Tesis presentada en opción al grado de Doctor en Ciencias Agrícolas. Instituto Nacional de Ciencias Agrícolas. La Habana, 2000.

INSMET. 2012. Impacto del Cambio Climático y Medidas de Adaptación en Cuba. Informe presentado en el marco de la Segunda Comunicación Nacional de Cuba al Convenio Marco de

Naciones Unidas sobre Cambio Climático. ISBN 978-959-300-036-2.

INSMET. 2015. Base de datos meteorológicos $1981-2010$.

Larrahondo, J.E, Villegas, F. Control y características de maduración. En CENICAÑA: El cultivo de la caña en la zona azucarera de Colombia.

Cali, CENICAÑA, 1995.p.297 - 313.

Lecha, L. B.; Paz, L. R. y Lapinel, B. 1994. El clima de Cuba, Edit. Academia, La Habana,

pp 186. ISBN 958-02-

000-0. Pérez Suárez, R; González

García, I.

Octubre del 2009.Temperatura superficial del aire. Capítulo 3 en Segunda Comunicación de Cuba a la Convención Marco de Naciones Unidas sobre

CambioClimático:

Actualizacióndel

conocimientosobre

variaciones, cambios y tendencias del clima en Cuba. Instituto de Meteorología.

Ponce-Hernández R.; Koohafkan P. y Antoine J. 2004. "Assessing carbon stocks and modeling win-win scenarios of carbon sequestration through land-use changes," Food and
Agriculture, Organization of the United Nations, Rome.

Reynoso A. 1862. Ensayo sobre el cultivo de la caña de azúcar. Publicaciones azucareras, edición 1988. $152 \mathrm{pp}$.

Robertson F.A. y Thorburn P.J. 2007. Management of sugarcane harvest residues: consequences for soil carbon and nitrogen. Aust. J. Soil Res., 45 , p. $13-23$. https://doi.org/10.1071/SR06080

Servicio de Recomendación de Fertilizantes y Enmiendas (SERFE). Informe de balance de área 2015.

Solano, O. 2014.Curso de Agrometeorología. Instituto Superior de Tecnologías y Ciencias Aplicada (InSTEC)

van Dillewijn C. 1952. Botánica de la caña de azúcar. Edición Revolucionaria. Instituto del Libro. 460 pp. 
Copyright (c) 2016 Grethel L. Sieiro Miranda, Maribel González Hidal go, Miguel Á. Lozano Rodriguez

BlancaE. Raya Cruz, Miguel Á González Salas y Enrique Aguirre López.

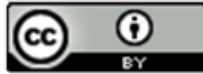

Este tex to está protegido por una licencia licencia Creative Commons 4.0.

Usted es libre para Compartir —copiar y redistribuir el material en cualquier medio o form ato- y Adaptar el documento - remezclar, transformar y crear a partir del material- para cualquier propósito, incluso para fines comerciales, siempre que cumpla la condición de:

Atribución: Usted debe dar crédito a la obra original de manera adecuada, proporcionar un enlace a la licencia, e in dicar si se han realizado cambios. Puede hacerlo en cualquier form a razonable, pero no de form a tal que sugiera que tiene el apoyo del licenciante o lo recibe por el uso que hace de la obra.

$\underline{\text { Resumendelicencia }}$ - Textocompletodelalicencia 